\title{
Solar System Effects in Schwarzschild Space Time
}

\author{
${ }^{1}$ Mst. Ayrin Aktar, ${ }^{2}$ Kamalesh Chandra Roy, ${ }^{3} \mathrm{Md}$. Al-Amin \\ I Lecturer, Department of Arts and Sciences, Bangladesh Army University of Science And Technology \\ (BAUST), Saidpur-5310, Nilphamari, Bangladesh) \\ ${ }^{2}$ (Associate Professor, Department of Mathematics, Begum Rokeya University, Rangpur-5400, Bangladesh) \\ ${ }_{3}^{3}$ (Lecturer, Department of Mathematics, Hajee Mohammad Danesh Science \& Technology University, \\ Dinajpur-5200, Bangladesh) \\ E-mail: ayrin.amath.ru@gmail.com
}

\begin{abstract}
Review works Solar System Effects in Schwarzschild de-Space Time. The Schwarzschild space-time describes the gravitational field of a spherically symmetric mass. Based on this space-time we calculate Solar system effects like gravitational redshift, light deflection, gravitational time delay, perihelion shift, geodetic precession.
\end{abstract}

Keywords: Schwarzschild Space-time, gravitational redshift, light deflection, gravitational time delay, perihelion shift, geodetic precession.

\section{Introduction}

Until now all gravitational effects in the Solar system and in binary systems are well described by means of the Einstein general theory of relativity [2][3]. Dark matter is introduced to obtain the gravitational field needed to describe observations like the galactic rotation curves, gravitational lensing, or the structure of the cosmic microwave background. Dark energy is invoked to explain the observed accelerated expansion of the universe by means of an additional energy momentum component. In general relativity, the repulsion necessary to obtain an accelerated expansion of the universe can be provided by the inclusion of a vacuum energy. Instead of postulating unknown forms of matter and energy another line of resolving these cosmological issues consists of a modification of e gravitational field. We present in the Schwarzschild space-time, on a Doppler measurement, used to determine the velocity of the Pioneer 10 and 11 spacecraft. We like to emphasize that the observational basis of the pioneer anomaly is by far not of the same quality as the Solar system effects. Therefore, we are looking for effects related to a space-time metric.

\section{Schwarzschild Space-time}

We calculate Solar system effects for the spherically symmetric Schwarzschild metric[11]

$d s^{2}=\alpha(r) d t^{2}-\alpha^{-1}(r) d r^{2}-r^{2}\left(d \theta^{2}+\sin ^{2} \theta d \Phi^{2}\right)$

Where,

$\alpha(r)=1-\frac{2 M}{r}$

and $\mathrm{M}$ the mass of the source. It is an exterior solution of the Einstein field equations for a spherical mass distribution

$$
R_{\mu v}-\frac{1}{2} g_{\mu v} R=8 \pi G T_{\mu v}
$$

\section{Gravitational Redshifst}

Since Schwarzschild is a stationary space-time there is a time like Killing vector so that in coordinates adapted to the symmetry the ratio of the measured frequency of a light ray crossing different positions is given by

$$
\begin{aligned}
\frac{v}{v_{0}}=\sqrt{\frac{g^{00}(r)}{g^{00}\left(r_{0}\right)}} \begin{array}{l}
\text { Where, } \\
g_{00}^{(0)}=1-\frac{2 M}{r}
\end{array}
\end{aligned}
$$

For small masses $\mathrm{M}$ this simplifies to

$$
\frac{v}{v_{0}}=1-\frac{M}{r}+\frac{M}{r_{0}}
$$

\section{Deflection of Light}

We choose the motion of point particles and light rays in the Schwarzschild space-time is described by the Lagrangian

$2 f=\alpha(r) \dot{t}^{2}-\alpha^{-1}(r) \dot{r}^{2}-r^{2} \dot{\phi}^{2}$ 
Where, the dot stands for differentiation with respect to the affine parameter $\lambda$, and where from the very beginning the motion is restricted to the $\theta=\frac{\pi}{2}$ plane. Since the metric (1.1) is independent of $t$ and $\phi$ the quantities

$E \equiv \alpha(r) \frac{d t}{d \lambda}, L \equiv r^{2} \frac{d \phi}{d \lambda}$,

Are conserved along the orbit of the particle and define its energy and angular momentum, respectively.

For light, the Lagrangian (1.4) vanishes. Multiplication of (1.4) by $\frac{\alpha}{L^{2}}$ and substitution of the angular momentum yields

$\frac{d \phi}{d r}= \pm \frac{1}{r^{2}}\left[\frac{1}{b^{2}}-\frac{\alpha(r)}{r^{2}}\right]^{-1 / 2}$

Where, $b \equiv \frac{L}{E}$ which in Schwarzschild space-time can be interpreted as impact parameter. The is related to increasing/decreasing $r$. The distance of closest approach \pm defined by

$\left.\frac{d \phi}{d r}\right|_{r=r_{0}}=\left[\frac{1}{b^{2}}-\frac{\alpha\left(r_{0}\right)}{r_{0}^{2}}\right]=0$

Relates $b$ to $r_{0}$. when we replace $b$ to $r_{0}$ in (1.6)

$\frac{d \phi}{d r}= \pm \frac{1}{r^{2}}\left[\frac{1}{r_{0}^{2}}\left(1-\frac{2 M}{r_{0}}\right)-\frac{1}{r^{2}}\left(1-\frac{2 M}{r}\right)\right]^{-1 / 2}$

\section{Gravitational Time Delay}

We consider the standard measurement of the time delay of light where a radar signal is sent from the Earth to pass close to the Sun and reflect off another planet or a spacecraft. Let $r_{\oplus}$ and $r_{R}$ be thedistance between the Sun and the Earth and the reflector, respectively. The time interval between emission and return of a pulse as measured by a clock on the Earth is

$\Delta T=2 t\left(r_{\oplus}, r_{0}\right)+2 t\left(r_{R}, r_{0}\right)$

Where, $t\left(r_{1}, r_{2}\right)$ is the elapsed coordinate time along the signal's trajectory between distances $r_{1}$ and $r_{2}$ to the Sun. The calculation of $t\left(r_{1}, r_{2}\right)$ requires to represent $t$ as a function of $r$ along the path of the pulse. From (1.5) and (1.6) we obtain

$\frac{d t}{d r}= \pm \frac{1}{a b}\left[\frac{1}{b^{2}}-\frac{\alpha(r)}{r^{2}}\right]^{-1 / 2}$

and, thus,

$\mathrm{t}\left(\mathrm{r}, r_{0}\right)=\int_{r_{0}}^{r} \frac{d r}{\alpha b(r)}\left[\frac{1}{b^{2}}-\frac{\alpha(r)}{r^{2}}\right]^{-1 / 2}$

Where, we can replace $b$ by $r_{0}$ using (1.7)

In the recent Cassini experiment [16] one has not measured the time delay but, instead, the relative change in the frequency

$y=\frac{v(t)-v_{0}}{v_{0}}=\frac{d}{d t} \Delta t$

Where, $v_{0}$ is the frequency of the radio waves emitted on the Earth.These radio waves reach Cassini with a frequency $v^{\prime}$ and are sent back with the same frequency $v^{\prime}$. The frequency of this signal reaching the Earth is $v(t)$.

\section{Perihelion Shift}

The perihelion is based on the modification of the geodesic equation with $u=\frac{1}{T}$ one obtains

$\frac{d^{2} u}{d \varphi^{2}}=\frac{M}{L^{2}}-u+3 M u^{2}$

Now we obtain 
$\delta \varphi=6 \pi \frac{G m}{a\left(1-\epsilon^{2}\right) c^{2}}$

\section{Geodesic Precession}

We want to calculate the geodesic precession by introducing a coordinate system rotating with angular velocity $\omega$.

We choose the new angular coordinate $\phi$ is as

$d \varphi=d \phi-\omega d t$

And gives in the equation plane $\theta=\pi / 2$ the metric

$d s^{2}=\left(\alpha-r^{2} \omega^{2}\right)\left(d t-\frac{r^{2} \omega}{\alpha-r^{2} \omega^{2}} d \varphi\right)^{2}-\alpha^{-1} d r^{2}-\frac{r^{2} \alpha}{\alpha-r^{2} \omega^{2}} d \varphi^{2}$

The canonical form of the metric is

$d s^{2}=e^{2 \Psi}\left(d t-\omega_{i} d x^{i}\right)^{2}-h_{i j} d x^{i} d x^{j}$

With

$e^{2 \Psi}=\alpha-r^{2} \omega^{2}, \omega_{1}=\omega_{2}=0, \omega_{3}=\frac{r^{2} \omega}{\alpha-r^{2} \omega^{2}}, h_{11}=\alpha^{-1}{ }_{j} h_{33}=\frac{r^{2} \alpha}{\alpha-r^{2} \omega^{2}}$

With the indices 1,2,3 referring to $r_{s} \theta, \varphi$ respectively. For a given $r=r_{0}$ we can choose an $\omega$ so that $\partial \Psi / \partial r=0$. since $\Psi$ is related to the acceleration ,this means that the line $r=r_{0}$ is geodesic. This corresponds to a modified Kepler frequency

$$
\omega^{2}=\frac{M}{r^{g}}
$$

Also obtained in[11] and implies

$$
\begin{aligned}
& e^{2 \Psi}=1-\frac{3 M}{r}, \\
& \omega_{1}=\omega_{2}=0, \\
& \omega_{3}=\frac{r^{2} \omega}{1-\frac{3 M}{r}}, \\
& h_{11}=\alpha^{-1}, \\
& h_{33}=\frac{r^{2} \alpha}{1-\frac{\mathrm{g} M}{r}}
\end{aligned}
$$

The rotation rate of a gyroscope at affixed position in the rotating coordinate system is given by[11]

$\Omega^{2}=\frac{e^{2 \Psi}}{8} h^{i k} h^{j l}\left(\omega_{i, j}-\omega_{j i}\right)\left(\omega_{k, l}-\omega_{l, k}\right)$

and points in the negative direction relative to the orbit. We obtain

$\Omega=\sqrt{\frac{M}{r^{s}}}$

\section{Doppler Tracking of Satellites on Escape Trajectories}

We will want to calculate the two-way Doppler tracking of a satellite moving on a geodesic radially away from the Sun. This amounts to an investigation of whether the presence of a cosmological constant might be made responsible for the anomalous acceleration observed for the Pioneer spacecraft[4]. For simplicity, we take an observer at a fixed radial distance from the Sun and a spacecraft moving radially .so that the Sun, the Earth and space craft lie on one line.

The measurement is carried out as follows: On the Earth a radio signal with frequency $v_{0}$ is sent to the spacecraft at position $r$,where it will arrive redshifted according to

$\tilde{v}=\sqrt{\frac{\alpha\left(r_{\ominus}\right)}{\alpha(r)}} v_{0}$

Due to the velocity of the spacecraft the measured is in addition Doppler shifted

$v^{v}=\frac{1}{\sqrt{1-\left(v^{r}\right)^{2}}}\left(1-v^{r}\right) \tilde{v}$ 
Where, $v^{r}=\theta^{r}(v) \quad$ the measured radial velocity ( $v$ is the 4-velocity of the spacecraft and $\theta^{r}$ is the normalized basis 1 -form in $r$-direction, $\quad g^{\mu v} \theta_{\mu}^{r} \theta_{v}^{r}=-1$; in coordinates, $\theta_{\mu}^{r}=\alpha^{-1 / 2}(0,1,0,0) \quad$. This is also the frequency sent back to Earth, where it arrives with the frequency

$v^{w}=\frac{1}{\sqrt{1-\left(v^{r}\right)^{2}}}\left(1-v^{r}\right) \sqrt{\frac{\alpha\left(r_{\theta}\right)}{\alpha(r)}} v^{2}$

The two-way Doppler tracking will then yield

$\frac{v^{\prime \prime}-v_{0}}{v_{0}}=-2 \frac{v^{r}}{1+v^{r}} \approx-2 v^{r}$

As expected, the gravitational redshift drops out. Only the motion of the spacecraft influences the result.

Therefore, what is finally needed is the solution for the radial velocity $v$ obtained from the geodesic equation

$0=\frac{d^{2} r}{d t^{2}}+\left(\left\{\begin{array}{c}r \\ \mu v\end{array}\right\}-\left\{\begin{array}{c}0 \\ \mu v\end{array}\right\} \frac{d r}{d t}\right) \frac{d x^{\mu}}{d t} \frac{d x^{v}}{d t}$

$=\frac{d^{2} r}{d t^{2}}-\frac{3}{2} \frac{1}{\alpha} \partial_{r} \alpha\left(\frac{d r}{d t}\right)^{2}+\frac{1}{2} \alpha \partial_{r} \alpha$

Where, $\left\{\begin{array}{c}r \\ \mu v\end{array}\right\}$ are the Christoffel symbols. We can safely neglect the term quadratic in the velocities. Then to first order in $M$

$\frac{d^{2} r}{d t^{2}}=-\frac{M}{r^{2}}$

This leads $\operatorname{to}_{2}^{1}\left(\frac{d r}{d t}\right)^{2}=E+\frac{M}{r}$

Where, $E$ is an integration constant.

The Doppler measurement in terms of the measured velocity is

$\frac{v^{n}-v_{0}}{v_{0}}=-2 \frac{v^{r}}{1+v^{r}} \approx-2=-2 \frac{d r / d t}{\sqrt{\alpha(r)}+d r / d t}$

We assume, $M / r \ll E$ and make an expansion with respect to $M \quad$.Furthermore,

$\frac{v^{n}-v_{0}}{v_{0}}=-2 \frac{\sqrt{2 E}}{1+\sqrt{2 E}}-\frac{2 M}{r} \frac{1+2 E}{(1+\sqrt{2 E})^{2} \sqrt{2 E}}$

Since $2 E$ corresponds to $v$ the non-relativistic limit is

$\frac{v^{n}-v_{0}}{v_{0}}=-2 \sqrt{2 E}-\frac{2 M}{r} \frac{1}{\sqrt{2 E}}$

For any non-Newtonian gravitational force the distance $r$ of the planetary orbits as function of the angle $\varphi$ is given by

$r(\varphi)=\frac{r_{0}(1+\epsilon)}{1+e \cos ((1+\epsilon) \varphi)}$

Where, $\epsilon_{\text {is }}$ the parameter related to the non-Newtonian part of the gravitational interaction describing the perihelion shift and the change in the planetary orbital parameter $r_{0} \quad$ (which is related to the semi-major axis). As a consequence, we can Newtonian or non-Einsteinian gravitational forces, there is an additional perihelion shift of the order $400 \mu$ as per century (which is the present day accuracy, then $\epsilon \approx 10^{-12}$ so that $\epsilon r_{0} \approx 15 \mathrm{~cm}$. This is clearly beyond any observational access for any planet in the Solar system.

\section{Conclusion}

We have calculated the Solar system effects in the frame of the Schwarzschild space-time. We considered the gravitational field of a spherically symmetric mass, described by the Schwarzschild space-time. We calculated how the absence of such a cosmological constant would affect Solar system effects like gravitational redshift,light deflection, gravitational time delay and geodetic precession. Comparing the theoretical results with the observations gives Solar system estimates on the magnitude of such an additional cosmological term in the Einstein equations. We conclude that the cosmic acceleration therefore does not lead to observable Solar system effects. 


\section{References}

[1]. Valeria Kagramanova , Jutta Kunz , Claus Lämmerzahl, Solar System Effects in Schwarzschild de-Space-Time, Institute of Nuclear Physics and Ulugh Beg Astronomical Institute, Astronomicheskaya 33, Tashkent 700052, Uzbekistan. Institut für Physik, Universität Oldenburg, Postfach 2503, D-26111 Oldenburg, Germany, ZARM, Universität Bremen, Am Fallturm, D-28359 Bremen, Germany, February 7, 2008.

[2]. C.M.Will. Theory and Experiment gravitational Physics(Revised) Cambridge University Press, Cambridge, 1993.

[3]. C.M.Will. The Confrontation between General Relativity and Experiment. Living Rev. Relativity, 2001,ww. Livingreviews.org/Irr2001-4

[4]. J.D. Anderson, P.A. Laing, E.L. Lau, A.S. Liu, M.M. Nieto, and S.G. Turyshev. Study of the anomalous acceleration of Pioneer 10 and 11. Phys. Rev., D 65:082004, 2002.

[5]. P.J.E. Peebles and B. Ratra. The cosmological constant and dark energy. Rev. Mod. Phys., 75:559, 2003.

[6]. C. Wetterich. Cosmologies with variable Newton's "constant". Nucl. Phys., B 302:645, 1988.

[7]. R.H. Sanders. Anti-gravity and galaxy rotation curves. Astron. Astrophys., 136:L21, 1984.

[8]. M. Milgrom. MOND - theoretical aspects. New Astr. Rev., 46:741, 2002.

[9]. D. Dvali, G. Gabadadze, and M. Porrati. 4D gravity on a brane in 5D Minkowski space. Phys. Lett., B 485:208, 2000.

[10]. H. Dittus and the Pioneer Explorer Collaboration. A mission to explore the Pioneer anomaly. In NN, editor, Trends in Space Science and Cosmic Vision 2030. ESA, Noordwijk, 2005; gr-qc/0506139.

[11]. W. Rindler. Relativity. Oxford University Press, Oxford, 2001.

[12]. A.W. Kerr, J.C. Hauck, and B. Mashhoon. Standard clocks, orbital precession and the cosmological constant. Class. Qauntum Grav., 20:2727, 2003.

[13]. R.F.C. Vessot, M.W. Levine, E.M. Mattison, E.L. Blomberg, T.E. Hoffmann, G.U. Nystrom, B.F. Farrel, R. Decher, P.B. Eby, C.R. Baughter, J.W. Watts, D.L. Teuber, and F.D. Wills.Test of relativistic gravitation with a space-borne hydrogen maser. Phys. Rev. Lett., 45:2081,1980.

[14]. K. Lake. Bending of light and the cosmological constant. Phys. Rev., D 65:087301, 2002

[15]. J.B. Hartle. Gravitation, An Introduction to Einstein's General Relativity. Addison Wesley,San Francisco, 2003.

[16]. B. Bertotti, L. Iess, and P. Tortora. A test of general relativity using radio links with the Cassini spacecraft. Nature, 425:374, 2003.

[17]. J.N. Islam. The cosmological constant and classical tests of general relativity. Phys. Lett., A 97:239, 1983.

[18]. G.V. Kraniotis and S.B. Whitehouse. Compact calculation of the perihelion precession of Mercury in general relativity, the cosmological constant and Jacobi's inversion problem. Class. Quantum Grav., 20:4817, 2003.

[19]. K. Nordtvedt. Improving gravity theory tests with solar system "grand fits". Phys. Rev., D 61:122001, 2001.

[20]. L. Iorio. Can solar system observations tell us something about the cosmological constant? gr-qc/0511137. To appear in Int. J. Mod. Phys. D.

[21]. Ph. Jetzer and M. Sereno. Two-body problem with the cosmological constant and observational constraints. astro-ph/0601612.

[22]. J.P. Mbelek. General relativity and quintessence explain the Pioneer anomaly. gr-qc/0402088.

[23]. M. Reuter and H. Weyer. Running newton constant, improved gravitational actions, and galaxy rotation curves. Phys. Rev., D 70:124028, 2004.

[24]. T. Damour, F. Piazza, and G. Veneziano. Runaway dilaton and equivalence principle violations. Phys. Rev. Lett., 89:081601, 2002.

[25]. T. Damour, F. Piazza, and G. Veneziano. Violations of the equivalence principle in a dilaton-runaway scenario. Phys. Rev., D 66:046007, 2002.

[26]. A. Lue. The phenomenology of Dvali-Gabadadze-Porrati cosmologies. Phys. Rep., 423:1, 2006

[27]. L. Iorio. On the effects of the Dvali-Gabadadze-Porrati braneworld gravity on the orbital motion of a test particle. Class. Quantum Grav., 22:5271, 2005.

[28]. L. Iorio. Secular increase of the Astronomical Unit and perihelion precessions as tests of the Dvali-Gabadadze-Porrati multidimensional braneworld scenario. JCAP, 0509:006, 2005. 